\title{
Optimization Problems of Costs in a MultiState System with Imperfect Maintenance
}

\author{
Soufiane Gasmi and Nizar Mannai \\ Tunis National Higher School of Engineering, University of Tunis, BP 56, 1008 Bab Menara, Tunisia \\ Correspondence should be addressed to Soufiane Gasmi; soufiane.kasmi3@gmail.com
}

Received 26 April 2013; Revised 19 December 2013; Accepted 27 December 2013; Published 27 February 2014

Academic Editor: Adiel Teixeira de Almeida

Copyright ( 2014 S. Gasmi and N. Mannai. This is an open access article distributed under the Creative Commons Attribution License, which permits unrestricted use, distribution, and reproduction in any medium, provided the original work is properly cited.

We consider optimal replacement policies with periodic imperfect maintenance actions and minimal repairs. The multistate system is minimally repaired at failure and imperfect maintenance actions are regularly carried out for preventive maintenance. The discrete modified Weibull distribution is introduced and some cost functions applied to this distribution are defined in order to be minimized. Moreover, we assume that the costs of preventive maintenance depend on the degree of repair via a Kijima type 2 model. For illustrative purpose, the obtained results are applied on sets of simulated data.

\section{Introduction}

In reliability theory, discrete failure data arise in several common situations. It is sometimes impossible to measure the life length of items with continuous scale. A discrete distribution is appropriate when we have a multistate system and the number of states prior to preventive maintenance is observed. A few results on discrete life distributions are introduced in the literature (Salvia and Bollinger [1], Kalbfleisch and Prentice [2], Shmueli et al. [3], and Nakagawa and Osaki [4]).

Recently, in reliability, much attention has been paid to the evaluation and application of multistate systems (MSS). We note that the MMS model provides a more flexible tool for modeling engineering systems in real life. This model is at first introduced in Barlow and Wu [5] and El-Neveihi et al. [6]. Some recent contributions on the subject are the works of (Zuo and Tian [7]; Li and Zuo [8]; Lisnianski and Ding [9]; Lisnianski and Frenkel [10]).

The most commonly used models for the failure process of a repairable system are known as perfect repair or as good as new and as minimal repair or as bad as old.

In the perfect repair case, each repair is perfect and leaves the system as if it were new; hence we obtain a renewal processes (RP). In the minimal repair case, each repair leaves the system in the same state as it was before failure, so we obtain nonhomogeneous Poisson processes (NHPP). It is well known in practice that the reality is between these two extreme cases. The repair may not yield a functioning item which is as good as new and the minimal repair assumption seems to be too pessimistic in repair strategies. From this it is seen that the imperfect repair is of great signification in practice.

Furthermore, the imperfect maintenance is a broader subject, in this way many authors have considered other aspects associated with the imperfect maintenance that corresponds to errors when doing the maintenance activities. In fact, when the maintainer makes a mistake it may correspond not only in terms of cost, but also in terms of reliability.

Currently, Berrade et al. [11] and Berrade et al. [12] have supposed that inspections are subject to error, and false positives or false negatives are possible. A false positive occurs when the inspection says the system is defective when in fact it is good. Consequently, the system is unnecessarily perfectly replaced and this implies an additional cost to the system owner. A false negative occurs when the inspection says the system is good when in fact it is defective. In this case, the system reliability will be reduced. 
In this paper, we are concerned with the modeling of an imperfect maintenance model; that is, the impact of preventive maintenance is between the two boundary cases: (a) minimal (as bad as old) and (b) perfect (as good as new).

Further, it is assumed that costs of preventive maintenance are not constant but depend on the degree of repair via a virtual age process.

A (MSS) with $N$ states is considered. On failure, corrective maintenance actions are minimal; that means that the item is minimally repaired at each failure and it will have the same state as just before failure. Therefore, we consider the inspection as a possible action from the maintenance. In this way we generalize the case that after preventive maintenance actions, only perfect repairs are considered.

In order to model the optimal maintenance actions, a new discrete modified Weibull distribution is introduced. At last some possible cost functions are proposed and optimal maintenance strategies are discussed.

\section{Modeling the Multistate System}

Most operating units (items) are repaired or replaced when they have failed. However, it may need much time and high cost to repair a failed item, so it is essential to maintain it in order to prevent failures and to reduce costs.

After failure the maintenance is called corrective maintenance (CM) and before failure it is called preventive maintenance (PM). Some PM policies and their optimization problems for shock and damage models are summarized in Nakagawa [13].

In practice, $\mathrm{PM}$ is used to lengthen the useful lifetime of items and to decrease average running cost by minimizing the occurrence of failures. Here we can note that most of the models concerning the modeling of repairable systems identify the minimal repair and the imperfect repair actions. Naturally, this popular assumption is a very unreal one.

In the following, a multistate system with $N$ states is considered. To simplify the notation and to use results from the imperfect maintenance models with discrete lifetime distribution, a simple time scale is suitable (Kahle [14]). Therefore, a time scale is introduced, and it will be assumed that in time 1 the item is in state one, in time 2 the item is in state two, and so forth. We remark that this time scale becomes more simple and can be generalized to any time scale. We studied the case that PM actions are regularly carried out. Furthermore, failures between PM actions are minimally repaired. The PM actions reset the failure rate proportional to the virtual age of the item. We assume that the failure rate at state, $k=1,2, \ldots, N$, is increasing in time $t$. In the case of multistate system, the effect of the PM actions is to reset the item in a lower state that means a younger state. We denote the state after PM actions by state $s$.

It is supposed that the reduction of the failure rate is done by using the concept of virtual age reduction introduced by Kijima [15] and Kijima et al. [16]. We will particularly use the Kijima's type 2 imperfect repair model to describe the PM actions. We note that the virtual age of the item at time $t_{k}$, following the repair, is defined by $V_{k}=\zeta_{k}\left(V_{k-1}+t_{k}-t_{k-1}\right)$, $k \geq 1$, where $t_{k}$ is time of the $k$ th sojourn and $\zeta_{k}\left(0 \leq \zeta_{k} \leq\right.$ 1 ) is the degree of repair at that time. We assume that $t_{0}=0$ and $V_{0}=0$. After repairing the failure intensity during the $(k+1)$ th sojourn is determined by $\lambda_{k+1}(t)=\lambda\left(t-t_{k}+V_{k}\right)$, $t_{k} \leq t<t_{k+1}, k \geq 0$.

The process defined by $\left(t, \zeta_{k}, k=1, \ldots, N\right)=t-t_{k}+V_{k}$, $t_{k} \leq t<t_{k+1}, k \geq 0$, is called the virtual age process. We can note that in Gasmi et al. [17] a generalized Kijima type 2 model was considered, where repairs give an additional impact made by using a major repair.

\section{The Discrete Modified Weibull Distribution}

In some situations, discrete lifetime distributions are appropriate to model lifetimes. The first discrete reliability distribution has been defined by Nakagawa and Osaki [4]. Furthermore, Kahle [14] studied some discrete lifetime distributions like the discrete uniform distribution, the Poisson distribution, and the truncated discrete Weibull distribution. In this paper the discrete modified Weibull distribution (DMWD) will be introduced.

We note that the modified Weibull distribution (MWD) is recently proposed by Sarhan and Zaindin [18]. This distribution is a general form for some most commonly used distributions in survival analysis such as exponential, Rayleigh, linear failure rate, and Weibull distribution. We will use the notation MWD $(\alpha, \beta, \gamma)$ to denote the modified Weibull distribution with the scale parameter $\alpha$ and the two shape parameters $\beta$ and $\gamma$.

Let $X$ be $\operatorname{MWD}(\alpha, \beta, \gamma)$ with density function:

$$
f(x, \theta)=\left(\alpha+\beta \gamma x^{\gamma-1}\right) \exp \left(-\alpha x-\beta x^{\gamma}\right), \quad x>0,
$$

where $\theta=(\alpha, \beta, \gamma), \gamma>0, \alpha, \beta \geq 0$, such that $\alpha+\beta>0$.

For the discretization of this distribution, we will put the probability mass of the interval $(k-1, k]$ into the point $k$; that is,

$$
\begin{aligned}
P(X=k)= & \int_{k-1}^{k} f(u) d u \\
= & \exp \left(-\alpha(k-1)-\beta(k-1)^{\gamma}\right) \\
& -\exp \left(-\alpha k-\beta k^{\gamma}\right), \quad k=1,2, \ldots
\end{aligned}
$$

The reliability function is given by the probability that the system is still alive at time $k$ :

$$
R(k)=P(X>k)=\sum_{i=k+1}^{\infty} P(X=i) ;
$$

therefore we have $P(X=k)=R(k-1)-R(k)$.

In the case of the DMWD we obtain $R(k)=\exp (-\alpha k-$ $\left.\beta k^{\gamma}\right), k=1,2, \ldots$.

The failure rate of a discrete distribution has been defined by Barlow et al. [19]:

$$
\lambda(k)=\frac{R(k-1)-R(k)}{R(k-1)}, \quad k=1,2, \ldots
$$




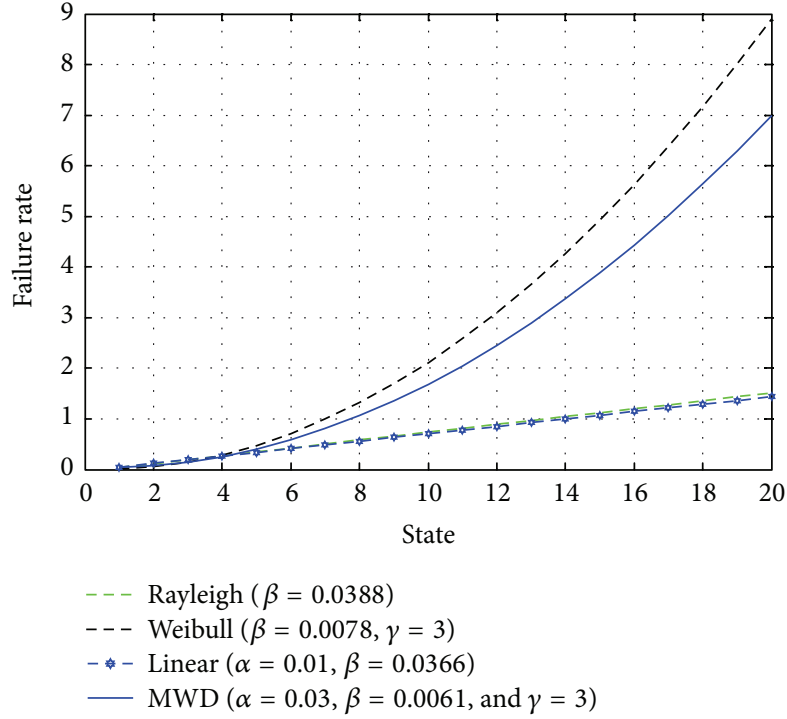

FIGURE 1: Discrete failure rates $r(k)$.

The definition of the failure rate as in (4) has many disadvantages. We cite that in the discrete case, the failure rate is bounded by 1 while most of continuous reliability models have unbounded failure rates. Moreover, $\lambda(k)$ is not a convex function. This restricts the interpretation of the failure rate in praxis. Besides, $\lambda(k)$ can never grow linearly or exponentially. Xie et al. [20] have shown that the cumulative hazard function, denoted by $H(k)$, verified the relation $H(k)=$ $-\ln R(k) \neq \sum_{i=1}^{k} \lambda(i)$.

They proposed a new redefined failure rate function for discrete distributions, denoted by

$$
r(k)=-\ln \frac{R(k)}{R(k-1)}=\ln \frac{R(k-1)}{R(k)} .
$$

Consequently, the above mentioned problems are resolved.

Both failure rates $\lambda(k)$ and $r(k)$ are linked by $r(k)=$ $-\ln (1-\lambda(k))$. On the other hand, we can see that the two failure rate definitions have the same monotonicity property.

Xie et al. [20] proved that if one is increasing/decreasing, the other is also increasing/decreasing. Therefore, the redefined failure rate function is more appropriate and is used for a much useful discrete reliability functions.

For the DMWD $(\alpha, \beta, \gamma)$, we have then

$$
\begin{array}{r}
r(k)=\left(\alpha k+\beta k^{\gamma}\right)-\left(\alpha(k-1)+\beta(k-1)^{\gamma}\right), \\
k=1,2, \ldots .
\end{array}
$$

The cumulative hazard function is defined by

$$
H(k)=\sum_{i=1}^{k} r(i)
$$

We obtain then the same form as for the continuous MWD.

$$
H(k)=\left(\alpha k+\beta k^{\gamma}\right), \quad k=1,2, \ldots
$$

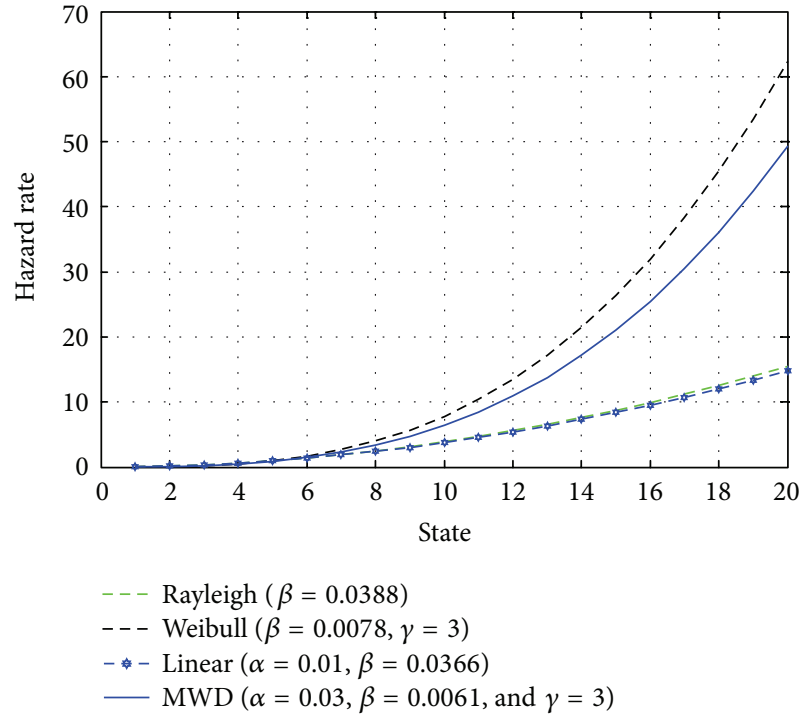

FIgUre 2: Cumulative hazard functions $H(k)$.

The $r$ th moment of the DMWD is given by $E\left(X^{r}\right)=$ $\sum_{i=1}^{\infty} i^{r}[R(i-1)-R(i)]$. If we put $r$ equal to 1 , we obtain $E(X)=$ $\sum_{i=0}^{\infty} \exp \left(-\alpha i-\beta i^{\gamma}\right)$ and the mean time to failure MTTF of the DMWD has then the form MTTF $=\sum_{i=0}^{\infty} \exp \left(-\alpha i-\beta i^{\gamma}\right)$. We can remark that in the case of $\beta=0$ we obtain the discrete exponential distribution with parameter $\alpha$. By putting $\alpha=0$ and $\gamma=2$ we obtain the discrete Rayleigh distribution with parameter $\beta$. If $\gamma=2$, a discrete linear failure rate with parameters $\alpha$ and $\beta$ is obtained. Finally if $\alpha=0$ the Weibull distribution with parameters $\beta$ and $\gamma$ will be derived.

Figures 1 and 2 show, respectively, different patterns of the discrete failure rate $r(k)$ and the cumulative hazard function $H(k)$ in the case of linear failure rate, Rayleigh distribution, Weibull distribution, and the modified Weibull distribution. We note that the parameters for all these distributions are chosen so that the expectation is equal to 5 and $N=20$.

Assumption 1. We suppose that the shape parameter $\gamma \geq 2$, this implies that $\lambda(k)$ is increasing and $H(k)$ is convex.

\section{The Maintenance Policy}

A sequential imperfect preventive maintenance policy was proposed in Nakagawa [21] and Chen et al. [22]. This policy supposes that PM is undertaken at fixed intervals.

We consider sequential PM policies for the system, where the distance between PM actions is always equal to $v$. Furthermore, all CM actions are minimal, that means that the item is minimally repaired at each failure and after repair the item has the same state as just before failure. It is assumed that the duration for $\mathrm{CM}$ and $\mathrm{PM}$ is negligible.

Following the strategy of Kahle [14], we assume that the multistate system has $N$ states. Let $s$ be the state of the item after such PM actions and the next PM executed $v$ states later. Every time when the item reaches the state $(s+v)$ the PM 
action resets it to the state $s$. In this case the following pairs of $v$ and $s$ are possible:

$$
\begin{gathered}
v=N-1, \quad s=1, \\
v=N-2, \quad s=1 \quad \text { or } \quad s=2, \ldots \\
\vdots \\
v=1, \quad s=1,2, \ldots, \quad \text { or } \quad s=N-1 .
\end{gathered}
$$

Let $\zeta=s /(s+v)$ be the degree of repair following the Kijima type 2 model. We have assumed that $0<\zeta<1$ and we eliminated the two cases:

(i) $\zeta=0$ which correspond to perfect repair action that involves that the item is reset to state $s=0$;

(ii) $\zeta=1$ which correspond to minimal repair action and engender $v=0$.

Now, our purpose is to obtain the pairs $v$ and $s$ minimizing the costs of PM and CM per time unit. In numerical examples, we investigate the effect of model parameters to the expected cost.

\section{The Optimal Expected Cost Function}

We consider maintenance policies for repairable systems in which the economical approach is our main objective. In our model each PM action reduces the age of the system to $s$ (state $s$ ) and every CM is minimal. The idea in some papers (Nakagawa [23]) assuming that each PM action has the same costs is not realistic. In Kahle [14] a new cost function which describes the cost of the PM action according to the degree of repair is introduced, where PM actions are undertaken at fixed times $v, 2 v, \ldots$.

Let $C_{\mathrm{CM}}$ and $C_{\mathrm{PM}}$ be, respectively, the cost of a corrective maintenance action and the cost of preventive maintenance action. The cost function per time unit is defined as follows:

$$
C_{\text {tot }}(s, v)=\frac{C_{\mathrm{PM}}(s, v)+C_{\mathrm{CM}}(H(s+v)-H(s))}{v} .
$$

Our main objective is to minimize this function with respect to $s$ and $v$. Now, we will introduce new defined cost functions applied to the DMWD.

5.1. The Costs of PM Action Proportional to the State before Repair. We assume that the cost function $C_{\mathrm{PM}}$ depends on the state just before the preventive maintenance action:

$$
C_{\mathrm{PM}}(s, v)=C_{s}\left(\frac{1}{s+v}\right)^{\delta},
$$

where $\delta \in \mathrm{IR}_{+}$has the role to make this cost function more flexible.

Let the ratio of costs $C_{\mathrm{CM}} / C_{s}=2, \delta=0.5$, and $N=20$. The cost function per time unit is plotted for the DMWD in Figure 3. We remark that the points will be connected with lines to make more visibility and that for different values of $v$.

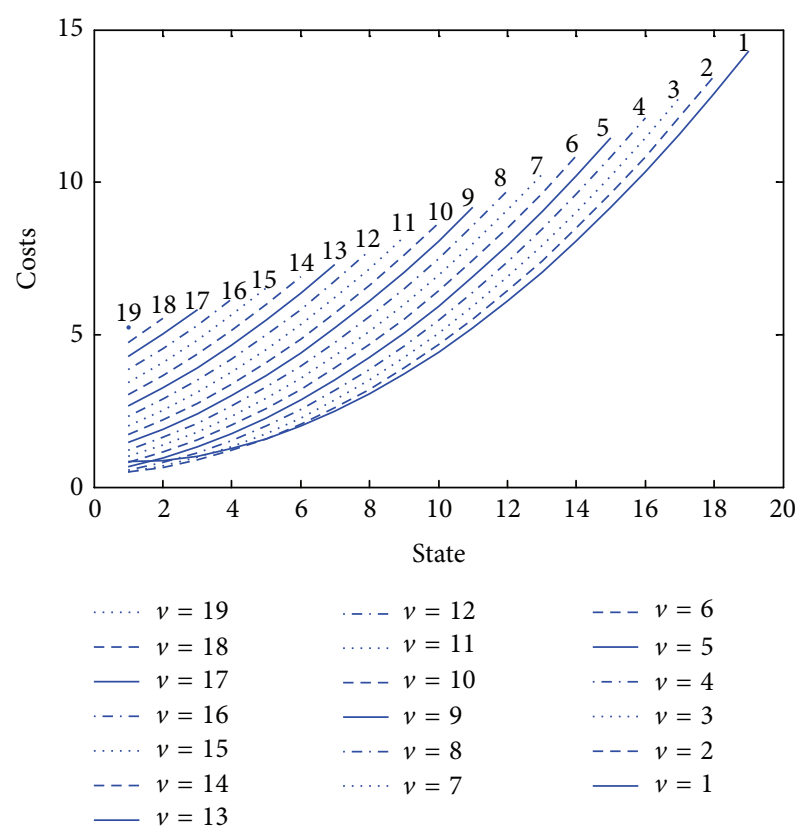

FIgURE 3: Cost function for DMWD $\left(\alpha=0.03, \beta=6.110^{-3}\right.$, and $\gamma=3)$.

Our cost function is convex; it follows then the uniqueness of the minimum.

In Table 1, we will present for different values of $\delta$ and different ratio costs the optimal states before the PM $(s+v)$ and after the PM $(s)$.

We have used the following discrete distributions: linear failure rate distribution (DLFRD), Rayleigh distribution (DRD), Weibull distribution (DWD), and the modified Weibull distribution (DMWD).

We also compared the cost function for these distributions and we noted that the parameters were chosen so that the expectation was the same for all distributions. Let this expectation be 5 .

5.2. The Costs of PM Action Proportional to the Impact of Repair. In this case, we will assume that the cost function $C_{\mathrm{PM}}$ depends only on the state after the PM action:

$$
C_{\mathrm{PM}}(s, v)=C_{I} \cdot\left(\frac{1}{s}\right)^{\delta},
$$

where $\delta \in \mathrm{IR}_{+}$.

Let the ratio of costs $C_{\mathrm{PM}} / C_{I}=2, \delta=2$, and $N=20$. In Figure 4, each line corresponds to a different value of $v$. This figure illustrates the cost function per time unit for the discrete modified Weibull distribution. As earlier, to make more visibility, the points are connected with lines. The states $(s+v)$ and $(s)$, corresponding to the unique optimum for the cost function, are given in Table 2 .

The cost functions for distributions used in Section 5.1 are compared. We note that all distributions have the same expectation 10 . 
TABLE 1: Optimal values for $s$ and $v$ and corresponding optimal costs $C_{\text {tot }}$.

\begin{tabular}{|c|c|c|c|c|}
\hline & $\begin{array}{c}\operatorname{DLFRD}(\alpha, \beta) \\
\alpha=0.01 \\
\beta=0.0366\end{array}$ & $\begin{array}{l}\operatorname{DRD}(\beta) \\
\beta=0.0388\end{array}$ & $\begin{array}{c}\operatorname{DWD}(\beta, \gamma) \\
\begin{array}{c}\beta=0.0078 \\
\gamma=3\end{array}\end{array}$ & $\begin{array}{c}\operatorname{DMWD}(\alpha, \beta, \gamma) \\
\alpha=0.03 \\
\beta=6.110^{-3} \\
\gamma=3\end{array}$ \\
\hline \multicolumn{5}{|l|}{$C_{\mathrm{CM}} / C_{s}=3$} \\
\hline$\delta=0.5$ & $\begin{array}{c}s=1 \\
v=3 \\
C_{\text {tot }}=0.7457\end{array}$ & $\begin{array}{c}s=1 \\
v=3 \\
C_{\text {tot }}=0.7485\end{array}$ & $\begin{array}{c}s=1 \\
v=2 \\
C_{\text {tot }}=0.5937\end{array}$ & $\begin{array}{c}s=1 \\
v=2 \\
C_{\text {tot }}=0.6182\end{array}$ \\
\hline$\delta=1$ & $\begin{array}{c}s=1 \\
v=2 \\
C_{\text {tot }}=0.6359\end{array}$ & $\begin{array}{c}s=1 \\
v=2 \\
C_{\text {tot }}=0.6321\end{array}$ & $\begin{array}{c}s=1 \\
v=2 \\
C_{\text {tot }}=0.4717\end{array}$ & $\begin{array}{c}s=1 \\
v=2 \\
C_{\text {tot }}=0.4962\end{array}$ \\
\hline$\delta=2$ & $\begin{array}{c}s=1 \\
v=2 \\
C_{\text {tot }}=0.5248\end{array}$ & $\begin{array}{c}s=1 \\
v=2 \\
C_{\text {tot }}=0.5210\end{array}$ & $\begin{array}{c}s=1 \\
v=2 \\
C_{\text {tot }}=0.3605\end{array}$ & $\begin{array}{c}s=1 \\
v=2 \\
C_{\text {tot }}=0.3851\end{array}$ \\
\hline$\delta=1 / 8$ & $\begin{array}{c}s=1 \\
v=3 \\
C_{\text {tot }}=0.8593\end{array}$ & $\begin{array}{c}s=1 \\
v=3 \\
C_{\text {tot }}=0.8621\end{array}$ & $\begin{array}{c}s=1 \\
v=2 \\
C_{\text {tot }}=0.7408\end{array}$ & $\begin{array}{c}s=1 \\
v=3 \\
C_{\text {tot }}=0.7572\end{array}$ \\
\hline$\delta=8$ & $\begin{array}{c}s=1 \\
v=1 \\
C_{\text {tot }}=0.3633\end{array}$ & $\begin{array}{c}s=1 \\
v=1 \\
C_{\text {tot }}=0.3530\end{array}$ & $\begin{array}{c}s=1 \\
v=1 \\
C_{\text {tot }}=0.1681\end{array}$ & $\begin{array}{c}s=1 \\
v=1 \\
C_{\text {tot }}=0.2229\end{array}$ \\
\hline \multicolumn{5}{|l|}{$C_{\mathrm{CM}} / C_{s}=2$} \\
\hline$\delta=0.5$ & $\begin{array}{c}s=1 \\
v=3 \\
C_{\text {tot }}=0.5527\end{array}$ & $\begin{array}{c}s=1 \\
v=3 \\
C_{\text {tot }}=0.5545\end{array}$ & $\begin{array}{c}s=1 \\
v=2 \\
C_{\text {tot }}=0.4920\end{array}$ & $\begin{array}{c}s=1 \\
v=3 \\
C_{\text {tot }}=0.4846\end{array}$ \\
\hline$\delta=1$ & $\begin{array}{c}s=1 \\
v=3 \\
C_{\text {tot }}=0.4694\end{array}$ & $\begin{array}{c}s=1 \\
v=3 \\
C_{\text {tot }}=0.4712\end{array}$ & $\begin{array}{c}s=1 \\
v=2 \\
C_{\text {tot }}=0.3700\end{array}$ & $\begin{array}{c}s=1 \\
v=2 \\
C_{\text {tot }}=0.3863\end{array}$ \\
\hline$\delta=2$ & $\begin{array}{c}s=1 \\
v=2 \\
C_{\text {tot }}=0.3684\end{array}$ & $\begin{array}{c}s=1 \\
v=2 \\
C_{\text {tot }}=0.3659\end{array}$ & $\begin{array}{c}s=1 \\
v=2 \\
C_{\text {tot }}=0.2589\end{array}$ & $\begin{array}{c}s=1 \\
v=2 \\
C_{\text {tot }}=0.2752\end{array}$ \\
\hline$\delta=1 / 8$ & $\begin{array}{c}s=1 \\
v=4 \\
C_{\text {tot }}=0.6637\end{array}$ & $\begin{array}{c}s=1 \\
v=3 \\
C_{\text {tot }}=0.6682\end{array}$ & $\begin{array}{c}s=1 \\
v=3 \\
C_{\text {tot }}=0.6087\end{array}$ & $\begin{array}{c}s=1 \\
v=3 \\
C_{\text {tot }}=0.5982\end{array}$ \\
\hline$\delta=8$ & $\begin{array}{c}s=1 \\
v=1 \\
C_{\text {tot }}=0.2435\end{array}$ & $\begin{array}{c}s=1 \\
v=1 \\
C_{\text {tot }}=0.2366\end{array}$ & $\begin{array}{c}s=1 \\
v=1 \\
C_{\text {tot }}=0.1134\end{array}$ & $\begin{array}{c}s=1 \\
v=1 \\
C_{\text {tot }}=0.1499\end{array}$ \\
\hline \multicolumn{5}{|c|}{$C_{\mathrm{CM}} / C_{s}=1$} \\
\hline$\delta=0.5$ & $\begin{array}{c}s=1 \\
v=4 \\
C_{\text {tot }}=0.3414\end{array}$ & $\begin{array}{c}s=1 \\
v=4 \\
C_{\text {tot }}=0.3445\end{array}$ & $\begin{array}{c}s=1 \\
v=3 \\
C_{\text {tot }}=0.3309\end{array}$ & $\begin{array}{c}s=1 \\
v=3 \\
C_{\text {tot }}=0.3256\end{array}$ \\
\hline$\delta=1$ & $\begin{array}{c}s=1 \\
v=3 \\
C_{\text {tot }}=0.2763\end{array}$ & $\begin{array}{c}s=1 \\
v=3 \\
C_{\text {tot }}=0.2773\end{array}$ & $\begin{array}{c}s=1 \\
v=3 \\
C_{\text {tot }}=0.2476\end{array}$ & $\begin{array}{c}s=1 \\
v=3 \\
C_{\text {tot }}=0.2423\end{array}$ \\
\hline$\delta=2$ & $\begin{array}{c}s=1 \\
v=2 \\
C_{\text {tot }}=0.2120\end{array}$ & $\begin{array}{c}s=1 \\
v=2 \\
C_{\text {tot }}=0.2107\end{array}$ & $\begin{array}{c}s=1 \\
v=2 \\
C_{\text {tot }}=0.1572\end{array}$ & $\begin{array}{c}s=1 \\
v=2 \\
C_{\text {tot }}=0.1654\end{array}$ \\
\hline$\delta=1 / 8$ & $\begin{array}{c}s=1 \\
v=5 \\
C_{\text {tot }}=0.4261\end{array}$ & $\begin{array}{c}s=1 \\
v=5 \\
C_{\text {tot }}=0.4314\end{array}$ & $\begin{array}{c}s=1 \\
v=3 \\
C_{\text {tot }}=0.4445\end{array}$ & $\begin{array}{c}s=1 \\
v=4 \\
C_{\text {tot }}=0.4248\end{array}$ \\
\hline$\delta=8$ & $\begin{array}{c}s=1 \\
v=1 \\
C_{\text {tot }}=0.1237\end{array}$ & $\begin{array}{c}s=1 \\
v=1 \\
C_{\text {tot }}=0.1203\end{array}$ & $\begin{aligned} s & =1 \\
v & =1 \\
C_{\text {tot }} & =0.0586\end{aligned}$ & $\begin{array}{c}s=1 \\
v=1 \\
C_{\text {tot }}=0.0769\end{array}$ \\
\hline
\end{tabular}


TABLE 2: Optimal values for $s$ and $v$ and corresponding optimal costs $C_{\text {tot }}$.

\begin{tabular}{|c|c|c|c|c|}
\hline & $\begin{array}{c}\operatorname{DLFRD}(\alpha, \beta) \\
\alpha=0.01 \\
\beta=0.0075\end{array}$ & $\begin{array}{l}\operatorname{DRD}(\beta) \\
\beta=0.0086\end{array}$ & $\begin{array}{c}\operatorname{DWD}(\beta, \gamma) \\
\beta=8.3110^{-4} \\
\gamma=3\end{array}$ & $\begin{array}{c}\operatorname{DMWD}(\alpha, \beta, \gamma) \\
\alpha=0.03 \\
\beta=4.7610^{-4} \\
\gamma=3\end{array}$ \\
\hline \multicolumn{5}{|l|}{$C_{\mathrm{PM}} / C_{I}=3$} \\
\hline$\delta=0.5$ & $\begin{array}{c}s=2 \\
v=6 \\
C_{\text {tot }}=0.3730\end{array}$ & $\begin{array}{c}s=1 \\
v=6 \\
C_{\text {tot }}=0.3727\end{array}$ & $\begin{array}{c}s=2 \\
v=4 \\
C_{\text {tot }}=0.3065\end{array}$ & $\begin{array}{c}s=2 \\
v=5 \\
C_{\text {tot }}=0.3273\end{array}$ \\
\hline$\delta=1$ & $\begin{array}{c}s=2 \\
v=5 \\
C_{\text {tot }}=0.3326\end{array}$ & $\begin{array}{c}s=2 \\
v=4 \\
C_{\text {tot }}=0.3310\end{array}$ & $\begin{array}{c}s=2 \\
v=4 \\
C_{\text {tot }}=0.2547\end{array}$ & $\begin{array}{c}s=2 \\
v=5 \\
C_{\text {tot }}=0.2858\end{array}$ \\
\hline$\delta=2$ & $\begin{array}{c}s=3 \\
v=2 \\
C_{\text {tot }}=0.2657\end{array}$ & $\begin{array}{c}s=3 \\
v=2 \\
C_{\text {tot }}=0.2615\end{array}$ & $\begin{array}{c}s=3 \\
v=2 \\
C_{\text {tot }}=0.1778\end{array}$ & $\begin{array}{c}s=3 \\
v=2 \\
C_{\text {tot }}=0.2157\end{array}$ \\
\hline$\delta=1 / 8$ & $\begin{array}{c}s=1 \\
v=7 \\
C_{\text {tot }}=0.3755\end{array}$ & $\begin{array}{c}s=1 \\
v=6 \\
C_{\text {tot }}=0.3727\end{array}$ & $\begin{array}{c}s=1 \\
v=5 \\
C_{\text {tot }}=0.3073\end{array}$ & $\begin{array}{c}s=1 \\
v=7 \\
C_{\text {tot }}=0.3373\end{array}$ \\
\hline$\delta=8$ & $\begin{array}{c}s=2 \\
v=1 \\
C_{\text {tot }}=0.1465\end{array}$ & $\begin{array}{c}s=2 \\
v=1 \\
C_{\text {tot }}=0.1327\end{array}$ & $\begin{array}{c}s=2 \\
v=1 \\
C_{\text {tot }}=0.0513\end{array}$ & $\begin{array}{c}s=2 \\
v=1 \\
C_{\text {tot }}=0.1211\end{array}$ \\
\hline \multicolumn{5}{|l|}{$\overline{C_{\mathrm{PM}} / C_{I}=2}$} \\
\hline$\delta=0.5$ & $\begin{array}{c}s=2 \\
v=7 \\
C_{\text {tot }}=0.2861\end{array}$ & $\begin{array}{c}s=2 \\
v=6 \\
C_{\text {tot }}=0.2895\end{array}$ & $\begin{array}{c}s=2 \\
v=5 \\
C_{\text {tot }}=0.2529\end{array}$ & $\begin{array}{c}s=2 \\
v=6 \\
C_{\text {tot }}=0.2580\end{array}$ \\
\hline$\delta=1$ & $\begin{array}{c}s=3 \\
v=5 \\
C_{\text {tot }}=0.2518\end{array}$ & $\begin{array}{c}s=2 \\
v=5 \\
C_{\text {tot }}=0.2545\end{array}$ & $\begin{array}{c}s=2 \\
v=5 \\
C_{\text {tot }}=0.2114\end{array}$ & $\begin{array}{c}s=3 \\
v=4 \\
C_{\text {tot }}=0.2187\end{array}$ \\
\hline$\delta=2$ & $\begin{array}{c}s=3 \\
v=3 \\
C_{\text {tot }}=0.1921\end{array}$ & $\begin{array}{c}s=3 \\
v=3 \\
C_{\text {tot }}=0.1915\end{array}$ & $\begin{array}{c}s=3 \\
v=2 \\
C_{\text {tot }}=0.1371\end{array}$ & $\begin{array}{c}s=3 \\
v=3 \\
C_{\text {tot }}=0.1571\end{array}$ \\
\hline$\delta=1 / 8$ & $\begin{array}{c}s=1 \\
v=8 \\
C_{\text {tot }}=0.2951\end{array}$ & $\begin{array}{c}s=1 \\
v=8 \\
C_{\text {tot }}=0.2967\end{array}$ & $\begin{array}{c}s=1 \\
v=6 \\
C_{\text {tot }}=0.2615\end{array}$ & $\begin{array}{c}s=1 \\
v=8 \\
C_{\text {tot }}=0.2718\end{array}$ \\
\hline$\delta=8$ & $\begin{array}{c}s=2 \\
v=1 \\
C_{\text {tot }}=0.0990\end{array}$ & $\begin{array}{c}s=2 \\
v=1 \\
C_{\text {tot }}=0.0897\end{array}$ & $\begin{array}{c}s=2 \\
v=1 \\
C_{\text {tot }}=0.0355\end{array}$ & $\begin{array}{c}s=2 \\
v=1 \\
C_{\text {tot }}=0.0820\end{array}$ \\
\hline \multicolumn{5}{|l|}{$C_{\mathrm{PM}} / C_{I}=1$} \\
\hline$\delta=0.5$ & $\begin{array}{c}s=2 \\
v=10 \\
C_{\text {tot }}=0.1858\end{array}$ & $\begin{array}{c}s=2 \\
v=9 \\
C_{\text {tot }}=0.1901\end{array}$ & $\begin{array}{c}s=2 \\
v=7 \\
C_{\text {tot }}=0.1867\end{array}$ & $\begin{array}{c}s=2 \\
v=8 \\
C_{\text {tot }}=0.1775\end{array}$ \\
\hline$\delta=1$ & $\begin{array}{c}s=3 \\
v=7 \\
C_{\text {tot }}=0.1552\end{array}$ & $\begin{array}{c}s=3 \\
v=6 \\
C_{\text {tot }}=0.1586\end{array}$ & $\begin{array}{c}s=3 \\
v=5 \\
C_{\text {tot }}=0.1473\end{array}$ & $\begin{array}{c}s=3 \\
v=6 \\
C_{\text {tot }}=0.1413\end{array}$ \\
\hline$\delta=2$ & $\begin{array}{c}s=3 \\
v=4 \\
C_{\text {tot }}=0.1128\end{array}$ & $\begin{array}{c}s=3 \\
v=4 \\
C_{\text {tot }}=0.1136\end{array}$ & $\begin{array}{c}s=3 \\
v=3 \\
C_{\text {tot }}=0.0894\end{array}$ & $\begin{array}{c}s=4 \\
v=3 \\
C_{\text {tot }}=0.0952\end{array}$ \\
\hline$\delta=1 / 8$ & $\begin{array}{c}s=1 \\
v=12 \\
C_{\text {tot }}=0.1984\end{array}$ & $\begin{array}{c}s=1 \\
v=11 \\
C_{\text {tot }}=0.2025\end{array}$ & $\begin{array}{c}s=1 \\
v=8 \\
C_{\text {tot }}=0.2007\end{array}$ & $\begin{array}{c}s=1 \\
v=10 \\
C_{\text {tot }}=0.1934\end{array}$ \\
\hline$\delta=8$ & $\begin{array}{c}s=2 \\
v=1 \\
C_{\text {tot }}=0.0514\end{array}$ & $\begin{array}{c}s=2 \\
v=1 \\
C_{\text {tot }}=0.0468\end{array}$ & $\begin{array}{c}s=2 \\
v=1 \\
C_{\text {tot }}=0.0197\end{array}$ & $\begin{array}{c}s=2 \\
v=1 \\
C_{\text {tot }}=0.0430\end{array}$ \\
\hline
\end{tabular}


TABLE 3: Costs of large repairs.

\begin{tabular}{lccccc}
\hline$\delta$ & 2 & 3 & 4 & 8 & 20 \\
\hline$C_{\mathrm{PM}}(s, v)$ & $0.9203 * C_{\mathrm{PR}}$ & $0.8829 * C_{\mathrm{PR}}$ & $0.8470 * C_{\mathrm{PR}}$ & $0.7174 * C_{\mathrm{PR}}$ & $0.4359 * C_{\mathrm{PR}}$ \\
\hline
\end{tabular}

TABLE 4: Costs of small repairs.

\begin{tabular}{lccccc}
\hline$\delta$ & $1 / 2$ & $1 / 3$ & $1 / 4$ & $1 / 8$ & $1 / 20$ \\
\hline$C_{\mathrm{PM}}(s, v)$ & $0.4308 * C_{\mathrm{PR}}$ & $0.5704 * C_{\mathrm{PR}}$ & $0.6564 * C_{\mathrm{PR}}$ & $0.8101 * C_{\mathrm{PR}}$ & $0.9192 * C_{\mathrm{PR}}$ \\
\hline
\end{tabular}

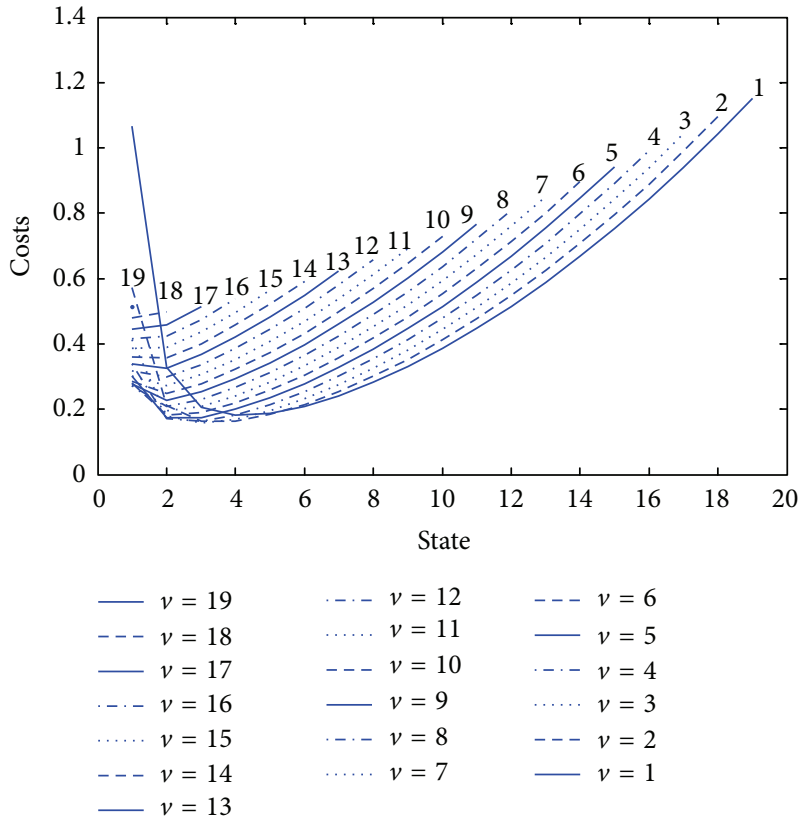

FIGURE 4: Cost function for DMWD $\left(\alpha=0.03, \beta=4.7610^{-4}\right.$, and $\gamma=3)$.

5.3. The Costs of PM Action Proportional to the Degree of Repair. Now, we consider a new cost function per time unit which depends on the degree of repair $\zeta$.

We know that for the Kijima type 2 model, the degree of repair $\zeta$ corresponds to $\zeta=s /(s+v)$.

Let $C_{\mathrm{PM}}(s, v)=C_{\mathrm{PR}} \cdot(1-(s /(s+v)) \exp (s /(s+v)-1))^{\delta}$ be the new cost function where $C_{\mathrm{PR}}$ are the perfect repair costs. This function can be well interpreted.

Remark 2. (a) For perfect repair, $\zeta=0$, implying that $(s=0)$, we obtain $C_{\mathrm{PM}}(s, v)=C_{\mathrm{PR}}$.

(b) For minimal repair, $\zeta=1$, involving that $(v=0)$, we obtain $C_{\mathrm{PM}}(s, v)=0$.

(c) If $\delta>1$, the cost function $C_{\mathrm{PM}}(s, v)$ is convex and with increasing $\delta$ the costs of large repair are relatively small.

(d) If $\delta<1$, the cost function $C_{\mathrm{PM}}(s, v)$ is concave and with decreasing $\delta$, the costs of relatively small repairs are close to that of perfect repair $C_{\mathrm{PR}}$.

Remark 3. (i) Let $\zeta=0.1$; Table 3 shows the costs of large repair for different values of $\delta$.

(ii) Let $\zeta=0.9$; Table 4 tells us about the costs of small repair for different values of $\delta$.

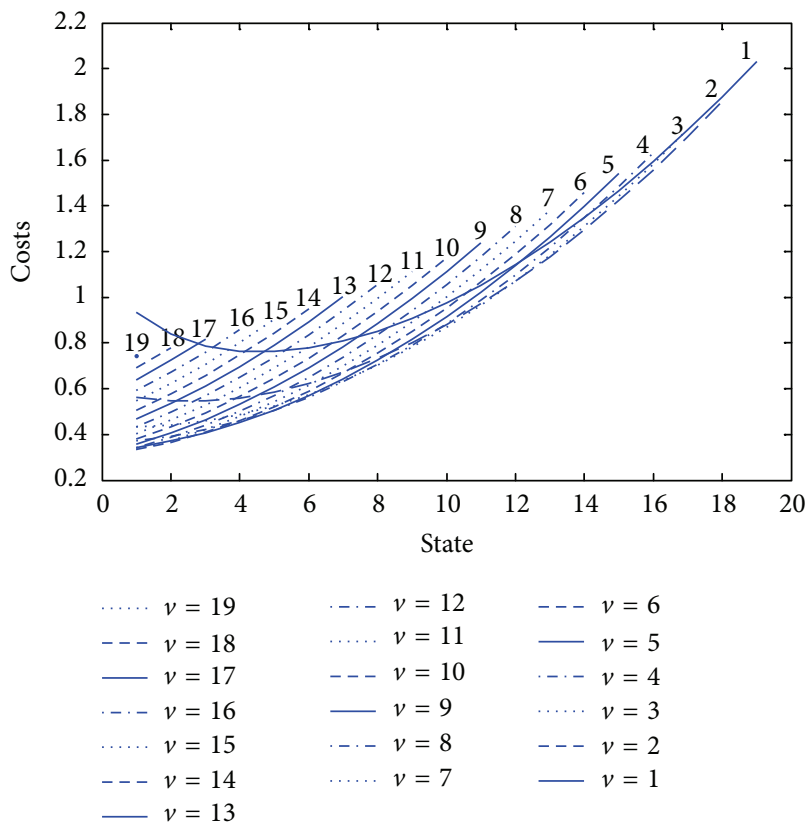

FIGURE 5: Cost function for DMWD $\left(\alpha=0.03, \beta=4.7610^{-4}\right.$, and $\gamma=3) ; \delta=0.5$.

The resulting cost function per time unit has then the following form:

$$
\begin{gathered}
C_{\text {tot }}(s, v)=\left(C_{\mathrm{PR}} \cdot\left(1-\left(\frac{s}{s+v}\right) \exp \left(\frac{s}{s+v}-1\right)\right)^{\delta}\right. \\
\left.+C_{\mathrm{CM}}(H(s+v)-H(s))\right) \times(v)^{-1} .
\end{gathered}
$$

This function should be minimized with respect to $s$ and $v$.

Similar to Sections 5.1 and 5.2, Figure 5 illustrates the cost function defined in (13) for the DMWD with $N$ states, $N=$ $20, C_{\mathrm{CM}} / C_{I}=3$, and $\delta=1 / 2$.

If $\delta=2$ and $C_{\mathrm{CM}} / C_{I}=3$, we obtain Figure 6. For each value of $v$, it can be seen that $C_{\mathrm{PM}}(s, v)$ has a unique minimum. The obtained results are given in Table 5 .

Once again, the choice of parameters of all used distributions in this table verified expectation 10 .

The summary of computation results is presented in Table 5. Hence, we obtain the following remark.

Remark 4. (a) If $\delta \leq 1$, then the state $s=1$ is always the optimal state after PM actions. This result is expected and can be interpreted as follows: the costs of PM actions are close to 
TABLE 5: Optimal values for $s$ and $v$ and corresponding optimal costs $C_{\text {tot }}$.

\begin{tabular}{|c|c|c|c|c|}
\hline & $\begin{array}{c}\operatorname{DLFRD}(\alpha, \beta) \\
\alpha=0.01 \\
\beta=5.410^{-3}\end{array}$ & $\begin{array}{c}\operatorname{DRD}(\beta) \\
\beta=8.610^{-3}\end{array}$ & $\begin{array}{c}\operatorname{DWD}(\beta, \gamma) \\
\beta=8.3910^{-4} \\
\gamma=3\end{array}$ & $\begin{array}{c}\operatorname{DMWD}(\alpha, \beta, \gamma) \\
\alpha=0.03 \\
\beta=4.7610^{-4} \\
\gamma=3\end{array}$ \\
\hline \multicolumn{5}{|c|}{$C_{\mathrm{CM}} / C_{I}=3$} \\
\hline$\delta=0.5$ & $\begin{array}{c}s=1 \\
v=8 \\
C_{\text {tot }}=0.3755\end{array}$ & $\begin{array}{c}s=1 \\
v=6 \\
C_{\text {tot }}=0.3675\end{array}$ & $\begin{array}{c}s=1 \\
v=5 \\
C_{\text {tot }}=0.3009\end{array}$ & $\begin{array}{c}s=1 \\
v=6 \\
C_{\text {tot }}=0.3331\end{array}$ \\
\hline$\delta=1$ & $\begin{array}{c}s=1 \\
v=7 \\
C_{\text {tot }}=0.3724\end{array}$ & $\begin{array}{c}s=1 \\
v=6 \\
C_{\text {tot }}=0.3626\end{array}$ & $\begin{array}{c}s=1 \\
v=5 \\
C_{\text {tot }}=0.2938\end{array}$ & $\begin{array}{c}s=1 \\
v=6 \\
C_{\text {tot }}=0.3281\end{array}$ \\
\hline$\delta=2$ & $\begin{array}{c}s=4 \\
v=1 \\
C_{\text {tot }}=0.3461\end{array}$ & $\begin{array}{c}s=4 \\
v=1 \\
C_{\text {tot }}=0.3508\end{array}$ & $\begin{array}{c}s=3 \\
v=1 \\
C_{\text {tot }}=0.2661\end{array}$ & $\begin{array}{c}s=4 \\
v=1 \\
C_{\text {tot }}=0.2963\end{array}$ \\
\hline$\delta=1 / 8$ & $\begin{array}{c}s=1 \\
v=8 \\
C_{\text {tot }}=0.3776\end{array}$ & $\begin{array}{c}s=1 \\
v=6 \\
C_{\text {tot }}=0.3714\end{array}$ & $\begin{array}{c}s=1 \\
v=5 \\
C_{\text {tot }}=0.3064\end{array}$ & $\begin{array}{c}s=1 \\
v=7 \\
C_{\text {tot }}=0.3363\end{array}$ \\
\hline$\delta=8$ & $\begin{array}{c}s=2 \\
v=1 \\
C_{\text {tot }}=0.1772\end{array}$ & $\begin{array}{c}s=1 \\
v=1 \\
C_{\text {tot }}=0.1328\end{array}$ & $\begin{array}{c}s=2 \\
v=1 \\
C_{\text {tot }}=0.0534\end{array}$ & $\begin{array}{c}s=2 \\
v=1 \\
C_{\text {tot }}=0.1227\end{array}$ \\
\hline \multicolumn{5}{|l|}{$C_{\mathrm{CM}} / C_{I}=2$} \\
\hline$\delta=0.5$ & $\begin{array}{c}s=1 \\
v=9 \\
C_{\text {tot }}=0.2886\end{array}$ & $\begin{array}{c}s=1 \\
v=7 \\
C_{\text {tot }}=0.2936\end{array}$ & $\begin{array}{c}s=1 \\
v=6 \\
C_{\text {tot }}=0.2572\end{array}$ & $\begin{array}{c}s=1 \\
v=7 \\
C_{\text {tot }}=0.2687\end{array}$ \\
\hline$\delta=1$ & $\begin{array}{c}s=1 \\
v=9 \\
C_{\text {tot }}=0.2864\end{array}$ & $\begin{array}{c}s=1 \\
v=7 \\
C_{\text {tot }}=0.2899\end{array}$ & $\begin{array}{c}s=1 \\
v=6 \\
C_{\text {tot }}=0.2522\end{array}$ & $\begin{array}{c}s=1 \\
v=7 \\
C_{\text {tot }}=0.2650\end{array}$ \\
\hline$\delta=2$ & $\begin{array}{c}s=5 \\
v=1 \\
C_{\text {tot }}=0.2666\end{array}$ & $\begin{array}{c}s=4 \\
v=1 \\
C_{\text {tot }}=0.2735\end{array}$ & $\begin{array}{c}s=4 \\
v=1 \\
C_{\text {tot }}=0.2214\end{array}$ & $\begin{array}{c}s=5 \\
v=1 \\
C_{\text {tot }}=0.2336\end{array}$ \\
\hline$\delta=1 / 8$ & $\begin{array}{c}s=1 \\
v=10 \\
C_{\text {tot }}=0.2902\end{array}$ & $\begin{array}{c}s=1 \\
v=8 \\
C_{\text {tot }}=0.2959\end{array}$ & $\begin{array}{c}s=1 \\
v=6 \\
C_{\text {tot }}=0.2610\end{array}$ & $\begin{array}{c}s=1 \\
v=8 \\
C_{\text {tot }}=0.2711\end{array}$ \\
\hline$\delta=8$ & $\begin{array}{c}s=2 \\
v=1 \\
C_{\text {tot }}=0.1200\end{array}$ & $\begin{array}{c}s=2 \\
v=1 \\
C_{\text {tot }}=0.0914\end{array}$ & $\begin{array}{c}s=2 \\
v=1 \\
C_{\text {tot }}=0.0374\end{array}$ & $\begin{array}{c}s=2 \\
v=1 \\
C_{\text {tot }}=0.0837\end{array}$ \\
\hline \multicolumn{5}{|l|}{$C_{\mathrm{CM}} / C_{I}=1$} \\
\hline$\delta=0.5$ & $\begin{array}{c}s=1 \\
v=13 \\
C_{\text {tot }}=0.1875\end{array}$ & $\begin{array}{c}s=1 \\
v=11 \\
C_{\text {tot }}=0.2010\end{array}$ & $\begin{array}{c}s=1 \\
v=8 \\
C_{\text {tot }}=0.1985\end{array}$ & $\begin{array}{c}s=1 \\
v=10 \\
C_{\text {tot }}=0.1916\end{array}$ \\
\hline$\delta=1$ & $\begin{array}{c}s=1 \\
v=13 \\
C_{\text {tot }}=0.1864\end{array}$ & $\begin{array}{c}s=1 \\
v=10 \\
C_{\text {tot }}=0.1993\end{array}$ & $\begin{array}{c}s=1 \\
v=8 \\
C_{\text {tot }}=0.1957\end{array}$ & $\begin{array}{c}s=1 \\
v=9 \\
C_{\text {tot }}=0.1895\end{array}$ \\
\hline$\delta=2$ & $\begin{array}{c}s=7 \\
v=1 \\
C_{\text {tot }}=0.1636\end{array}$ & $\begin{array}{c}s=6 \\
v=1 \\
C_{\text {tot }}=0.1776\end{array}$ & $\begin{array}{c}s=5 \\
v=1 \\
C_{\text {tot }}=0.1632\end{array}$ & $\begin{array}{c}s=6 \\
v=1 \\
C_{\text {tot }}=0.1566\end{array}$ \\
\hline$\delta=1 / 8$ & $\begin{array}{c}s=1 \\
v=14 \\
C_{\text {tot }}=0.1883\end{array}$ & $\begin{array}{c}s=1 \\
v=11 \\
C_{\text {tot }}=0.2021\end{array}$ & $\begin{array}{c}s=1 \\
v=8 \\
C_{\text {tot }}=0.2006\end{array}$ & $\begin{array}{c}s=1 \\
v=10 \\
C_{\text {tot }}=0.1930\end{array}$ \\
\hline$\delta=8$ & $\begin{array}{c}s=2 \\
v=1 \\
C_{\text {tot }}=0.0628\end{array}$ & $\begin{array}{c}s=2 \\
v=1 \\
C_{\text {tot }}=0.0485\end{array}$ & $\begin{array}{c}s=2 \\
v=1 \\
C_{\text {tot }}=0.0215\end{array}$ & $\begin{array}{c}s=2 \\
v=1 \\
C_{\text {tot }}=0.0446\end{array}$ \\
\hline
\end{tabular}




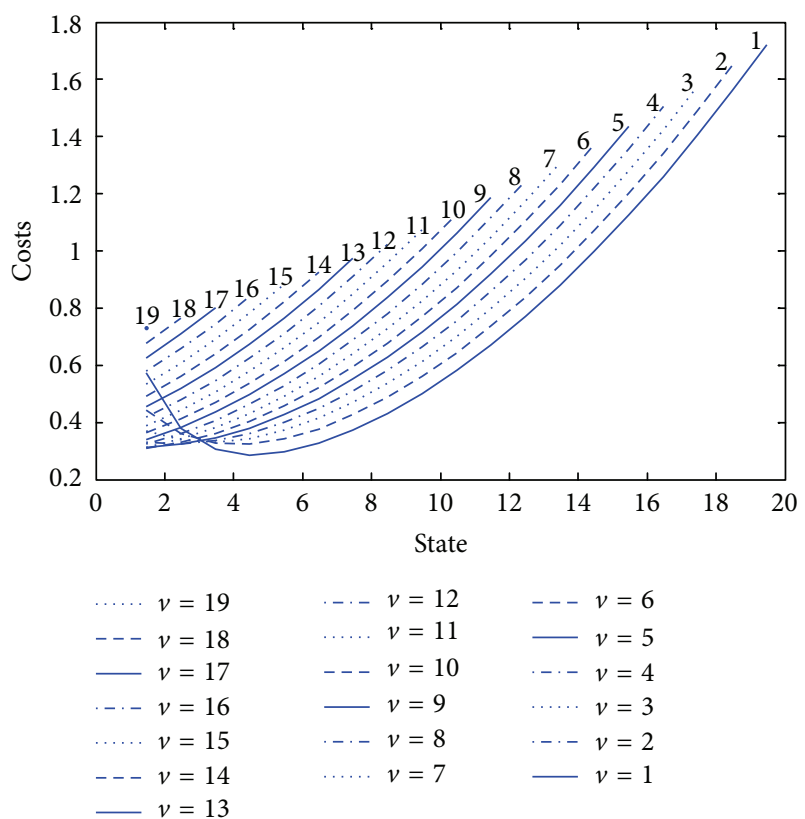

Figure 6: Cost function for DMWD $\left(\alpha=0.03, \beta=4.7610^{-4}\right.$, and $\gamma=3) ; \delta=2$.

the costs of perfect repair $C_{\mathrm{PR}}$; this implies that the optimal policy will be a perfect repair strategy.

(b) If $\delta>1$, then $v$ is always equal to 1 and the optimal distance between maintenance is short. This result can be interpreted as follows: the optimum for the cost function is obtained for relatively small repairs.

(c) If the failure rate is DMWD or DWD the obtained results are quite similar.

(d) If the failure rate is that of DLFRD, then the optimal distance between PM actions is larger than that for the rest failure rates. small.

(e) If $\delta=8$, then the optimal cost per time unit is very

(f) In the case of DMWD with parameters $\alpha=0.03, \beta=$ $4.7610^{-4}$, and $\gamma=3$, If $C_{\mathrm{CM}} / C_{I}=3$ and $\delta=1 / 2$, we obtain then the unique optimum for both $s=1$ and $v=6$. This implies the repair degree $\zeta=1 / 7$ and the total costs are $C_{\text {tot }}=$ 0.3331 .

(g) If we use the same parameters as in (f) and after replacing $\delta$ by 8 , we obtain then the unique optimum in $s=2$ and $v=1$. It follows then that $\zeta=2 / 3$ and $C_{\text {tot }}=0.1227$.

\section{Conclusions}

We considered an incomplete maintenance model; that is, the impact of PM is not minimal (ABAO) and not perfect (AGAN) but lies between these boundary cases. The PM actions reset the failure rate of the item proportional to the virtual age. We assumed that the reduction of the failure rate is done by using the Kijima's type 2 virtual age model. Further, all CM actions are minimal, which means that the item is minimally repaired at each failure.

In this research, the costs of PM actions are not constant but depend on (a) the state of the item just before the PM actions, (b) on the impact of repair, and (c) on the degree of repair. We note that the last case is very realistic and the obtained function can be well interpreted in praxis. Furthermore a periodic replacement policy is studied. We discussed the discrete failure data and the situations where the use of a continuous scale in order to measure the life length is quite artificial, so a DMWD is considered to describe the failure of the item under study.

The expected total optimal maintenance cost under discrete modified Weibull distribution (DMWD) is obtained. It is shown that the optimum for the cost function is unique.

Furthermore, we can consider models with imperfect repairs at failures. We expect that PM models will be applied to real systems and some results to these subjects will be studied in near future.

\section{Conflict of Interests}

The authors declare that there is no conflict of interests regarding the publication of this paper.

\section{Acknowledgments}

The authors would like to thank the editor and the anonymous reviewers for their invaluable comments and suggestions to improve the quality of the paper.

\section{References}

[1] A. A. Salvia and R. C. Bollinger, "On discrete hazard functions," IEEE Transactions on Reliability, vol. R-31, no. 5, pp. 458-459, 1982.

[2] J. D. Kalbfleisch and R. L. Prentice, The Statistical Analysis of Failure Time Data, John Wiley \& Sons, New York, NY, USA, 1980.

[3] G. Shmueli, T. P. Minka, J. B. Kadane, S. Borle, and P. Boatwright, "A useful distribution for fitting discrete data: revival of the Conway-Maxwell-Poisson distribution," Journal of the Royal Statistical Society C, vol. 54, no. 1, pp. 127-142, 2005.

[4] T. Nakagawa and S. Osaki, "The discrete Weibull distribution," IEEE Transactions on Reliability, vol. R-24, no. 5, pp. 300-301, 1975.

[5] R. E. Barlow and A. S. Wu, "Coherent systems with multi-state components," Mathematics of Operations Research, vol. 3, no. 4, pp. 275-281, 1978.

[6] E. El-Neveihi, F. Proschan, and J. Setharaman, "Multi-state coherent systems," Journal of Applied Probability, vol. 15, pp. 675-688, 1978.

[7] M. J. Zuo and Z. Tian, "Performance evaluation of generalized multi-state k-out-of-n systems," IEEE Transactions on Reliability, vol. 55, no. 2, pp. 319-327, 2006.

[8] W. Li and M. J. Zuo, "Reliability evaluation of multi-state weighted k-out-of-n systems," Reliability Engineering and System Safety, vol. 93, no. 1, pp. 160-167, 2008.

[9] A. Lisnianski and Y. Ding, "Redundancy analysis for repairable multi-state system by using combined stochastic processes methods and universal generating function technique," Reliability Engineering and System Safety, vol. 94, no. 11, pp. 1788-1795, 2009. 
[10] A. Lisnianski and I. Frenkel, "Non-homogeneous markov reward model for aging multistate system under minimal repair," International Journal of Performability Engineering, vol. 5, no. 4, pp. 303-312, 2009.

[11] M. D. Berrade, C. A. V. Cavalcante, and P. A. Scarf, "Maintenance scheduling of a protection system subject to imperfect inspection and replacement," European Journal of Operational Research, vol. 218, no. 3, pp. 716-725, 2012.

[12] M. D. Berrade, P. A. Scarf, C. A. V. Cavalcante, and R. A. Dwight, "Imperfect inspection and replacement of a system with a defective state: a cost and reliability analysis," Reliability Engineering \& Systems Safety, vol. 120, pp. 80-87, 2013.

[13] T. Nakagawa, Shock and Damage Models in Reliability Theory, Springer, London, UK, 2007.

[14] W. Kahle, "Optimal incomplete maintenance in multi-state systems," in Recent Advances in System Reliability, pp. 209-218, Springer, London, UK, 2012.

[15] M. Kijima, "Some results for repairable systems with general repair," Journal of Applied Probability, vol. 26, pp. 89-102, 1989.

[16] M. Kijima, H. Morimura, and Y. Suzuki, "Periodical replacement problem without assuming minimal repair," European Journal of Operational Research, vol. 37, no. 2, pp. 194-203, 1988.

[17] S. Gasmi, C. E. Love, and W. Kahle, "A general repair, proportional-hazards, framework to model complex repairable systems," IEEE Transactions on Reliability, vol. 52, no. 1, pp. 2632, 2003.

[18] A. M. Sarhan and M. Zaindin, "Modified Weibull distribution," Applied Sciences, vol. 11, pp. 123-136, 2009.

[19] R. E. Barlow, A. W. Marshall, and F. Proschan, "Properties of probability distributions with monotone hazard rate," Annals of Mathematical Statistics, vol. 34, pp. 375-389, 1963.

[20] M. Xie, O. Gaudoin, and C. Bracquemond, "Redefining failure rate function for discrete distributions," International Journal of Reliability, Quality and Safety Engineering, vol. 9, no. 3, pp. 275285, 2002.

[21] T. Nakagawa, Advances Reliability Models and Maintenance Policies, Springer, London, UK, 2008.

[22] M. Chen, S. Mizutani, and T. Nakagawa, "Random and age replacement policies," International Journal of Reliability, Quality and Safety Engineering, vol. 17, no. 1, pp. 27-39, 2010.

[23] T. Nakagawa, "Imperfect preventive maintenance models," in Stochastic Models in Reliability and Maintenance, S. Osaki, Ed., Springer, Berlin, Germany, 2002. 

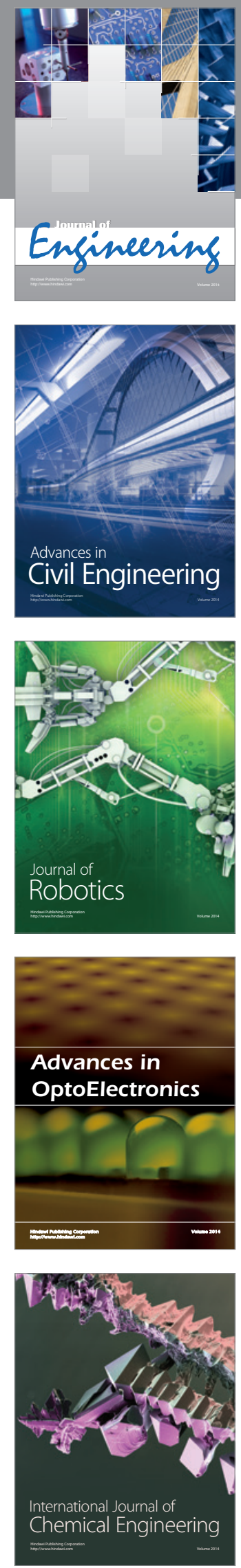

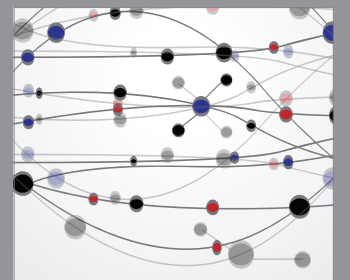

The Scientific World Journal
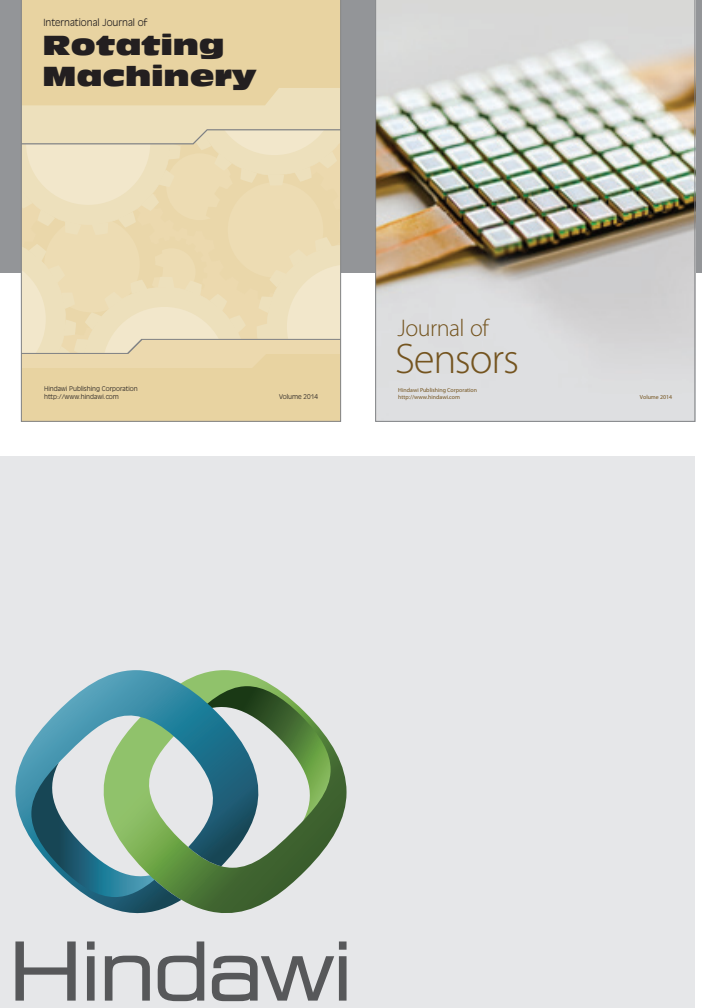

Submit your manuscripts at http://www.hindawi.com
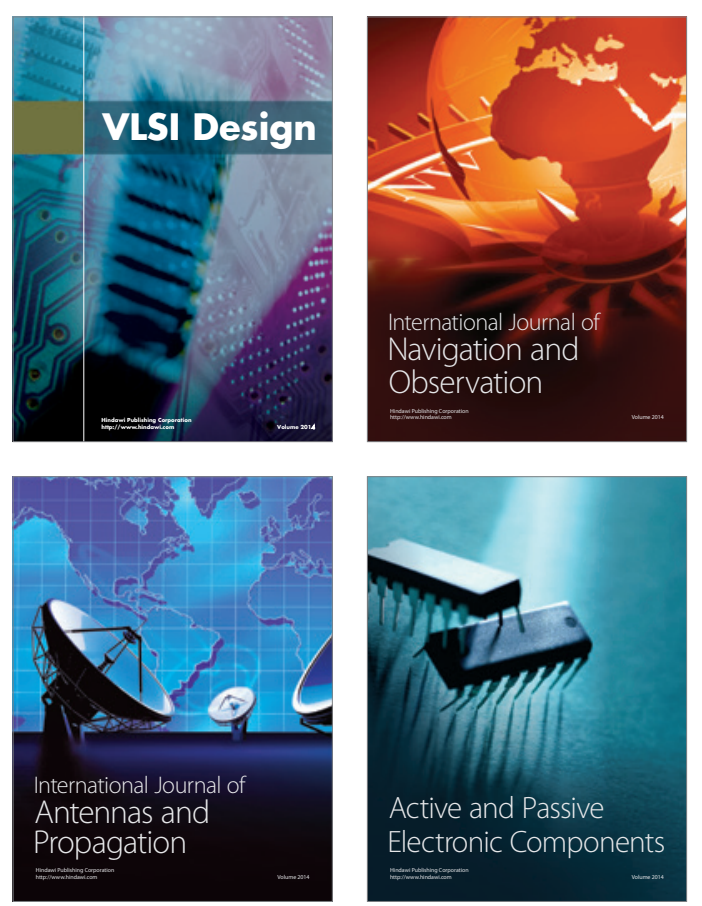
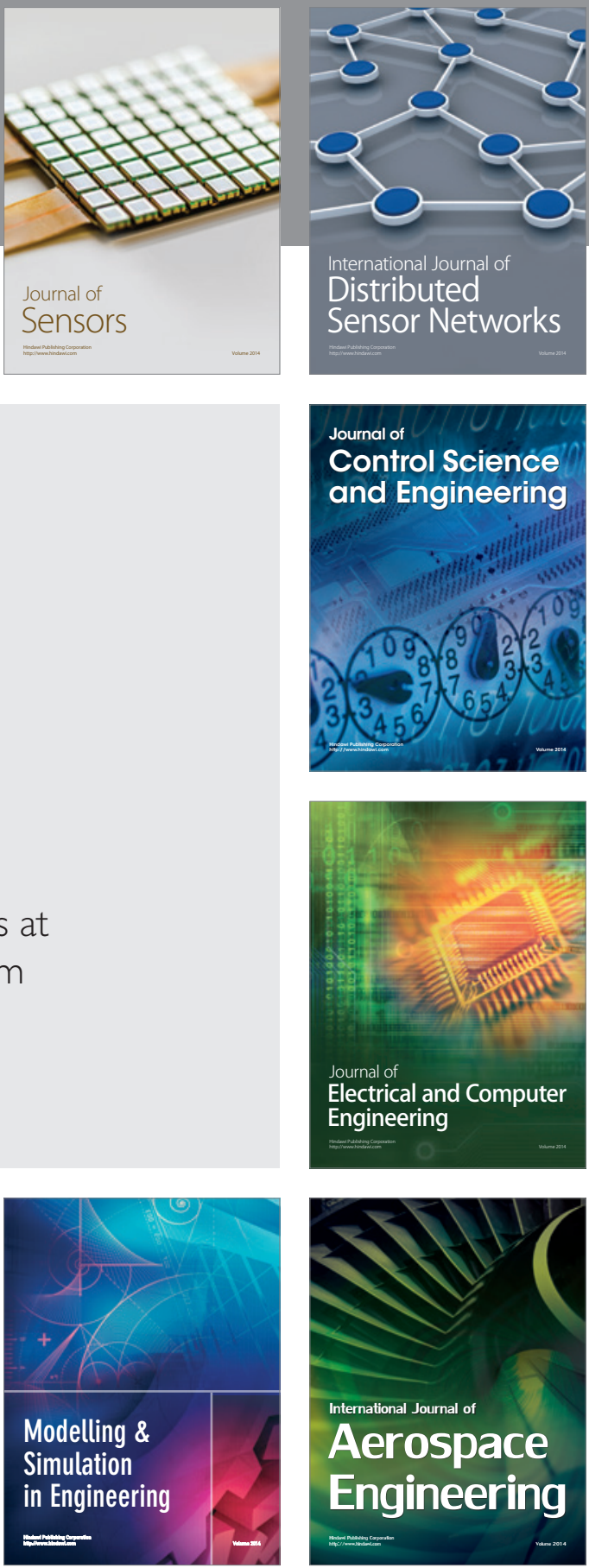

Journal of

Control Science

and Engineering
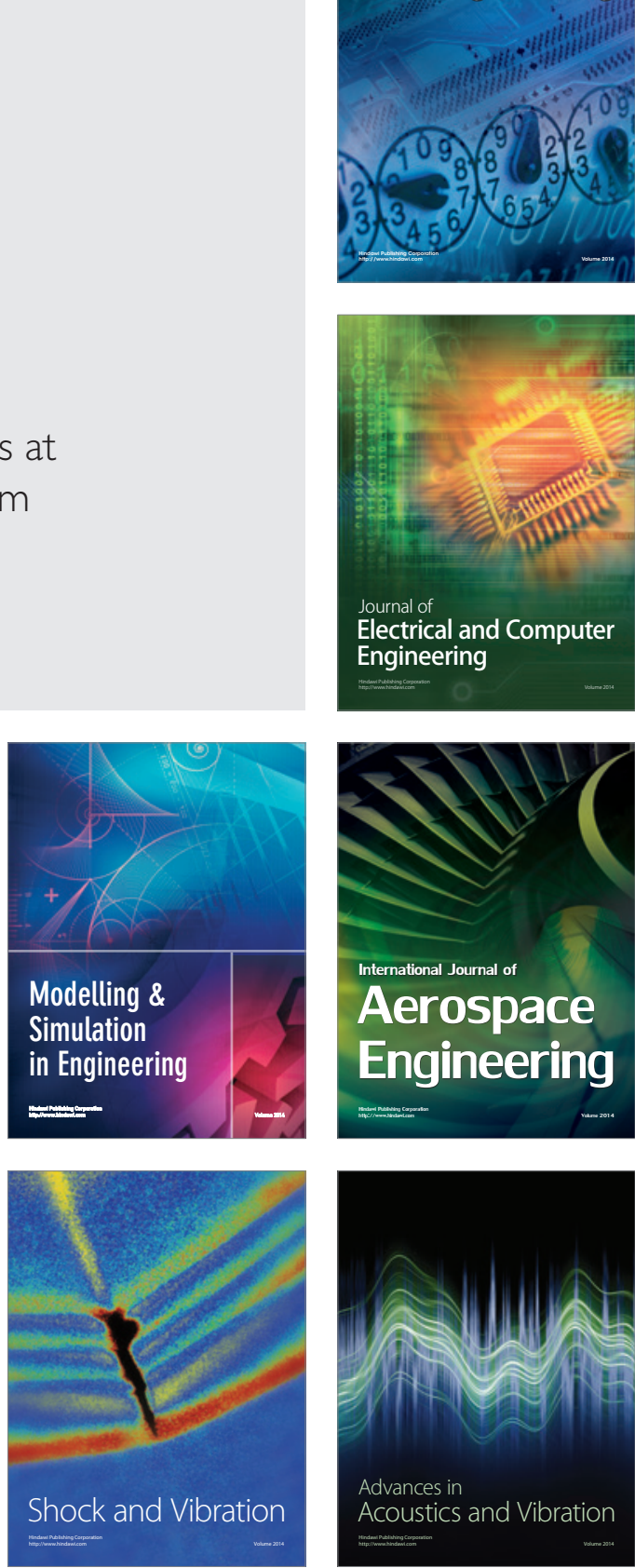